\title{
Paper Interference Management using Power Control for Device-to-Device Communication in Future Cellular Network
}

\author{
Toha Ardi Nugraha, Muhammad Putra Pamungkas, and Anna Nur Nazilah Chamim \\ Department of Electrical Engineering, Universitas Muhammadiyah Yogyakarta, Yogyakarta, Indonesia
}

\begin{abstract}
There are many scenarios that have been proposed for fifth generation (5G) networks. Some of them, if implemented, will bring fundamental changes at the architectural and node level. One example of such proposed technologies is device-to-device (D2D) communications which will change the nature of conventional cellular network design. D2D permits direct communication between two or more user devices without intervention of the base station (i.e. eNB). D2D can ensure network performance improvement over the traditional cellular network, because it can offload the mobile data traffic from the other devices. However, applying D2D features in a cellular network will bring about more complex interference problems, since D2D communication uses the same band as its underlying cellular communication network. The aim of this research is to investigate interference-related problems caused by D2D communications, affecting the underlying cellular networks, during downlink and uplink transmissions. The paper examines the use of power control methods to mitigate interference. A comparison is offered between fixed power level (FC) with or without power control, and adaptive power controls using two methods (AC1 and AC2), on a base station or on each of the D2D devices, based on the measured signal to interference plus noise ratio (SINR). The simulation results show that both power control methods contribute to improvement of network performance. $\mathrm{AC} 1$ and $\mathrm{AC} 2$ can improve SINR by about $1 \mathrm{~dB}$ and $0.5 \mathrm{~dB}$ compared to $\mathrm{FC}$ in a downlink transmission, and by $0.5 \mathrm{~dB}$ in an uplink transmission.
\end{abstract}

Keywords-cellular network, device-to-device communication, interference management, power control.

\section{Introduction}

There are many concepts, design criteria and scenarios that have been proposed for fifth generation $(5 \mathrm{G})$ cellular networks. Some of them, if implemented, will bring about fundamental changes at the architectural and node level. One example of such proposed technologies is device-todevice (D2D) communications [1]. D2D is a new feature for future cellular systems. In conventional cellular networks, each device or user equipment (UE) communicates directly with the base station (BS) via downlink (DL) and uplink (UL) paths [2]. The infrastructure of cellular networks will be changed in the new system. D2D permits direct communication between two user devices, without using the BS. D2D can improve performance of a traditional cellular network, due to D2D offloading the mobile data traffic from other devices.

Some researches has already been performed to investigate D2D communication in a cellular network [3]. Since no D2D standard has been proposed for $5 \mathrm{G}$ systems yet, the D2D model remains widely open [4]. However, D2D communications may results in cellular networks suffering from interference-related problems, as D2D communication shares the same frequency band as its underlying cellular network. Therefore, interference management aiming to reduce the adverse effects is crucial.

Many studies have been performed to investigate D2D's underlying cellular networks, and to assess various aspects related to coverage [5], [6]. One of the solutions adopted to manage interference-related problems is to separate the frequency allocation for D2D communications and macro cell communications, as presented in [7], [8]. However, limitation of the frequency spectrum is a common and serious problem, because of numerous devices staying on within a macro cell cellular network [9]. One of the solutions is to use a different frequency for each device, but this scheme is less effective, because in areas in which D2D communication is not used frequently, frequency domain allocated to D2D communications will be wasted.

In order to solve the frequency spectrum allocation problems, paper [10] proposes a shared/dedicated resource allocation method for D2D communications using underlying cellular networks. Therefore, some researchers have made proposed other solutions to manage the interference-related problems occurring within the same frequency spectrum for D2D communication, by implementing power control methods, e.g. [11]. This paper proposes a random network model for a D2D underlying cellular system using stochastic geometry, as well as developed centralized and distributed power control algorithms, but this method turned out to be very complex. Paper [12] investigated joint resource allocation and power control for D2D communications and the 
(a)

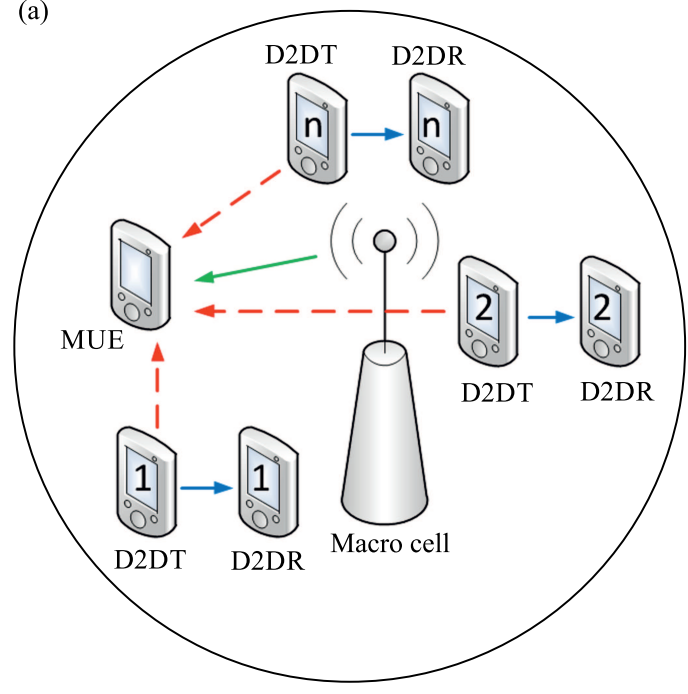

MUE: macro cell UE

D2D (T/R): device-to-device (transmitter/receiver)

No. of users: $1,2, \ldots, n$ (b)

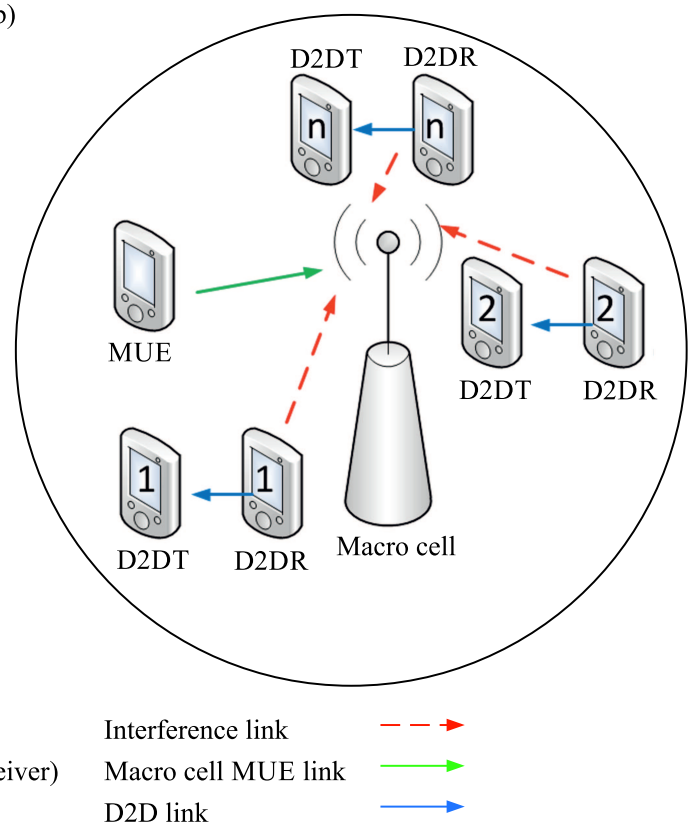

Fig. 1. Downlink and uplink transmissions of cellular network with D2D deployed in the single macro cell scenario: (a) first analyzed scenario, (b) second case.

underlying cellular networks, and the result focused on optimizing energy efficiency (EE) of D2D communications. The other method implemented the adaptive power control method [13]. In that paper, the simulation focused solely on DL transmissions. Another paper [14] also investigated D2D communication within an underlying cellular system, but only focused on UL transmissions.

The objective of this research is to manage interferencerelated problems in D2D communications and underlying cellular networks, affecting both types of transmissions, i.e. DL and UL. This research proposes the use of power control methods to mitigate interference in both directions. The methods compare two schemes, i.e. fixed power level (FC) and adaptive power controls (AC1 and $\mathrm{AC} 2$ ). The methods are implemented on BS or on D2D UE, based on the signal to interference plus noise ratio (SINR) measured in each device.

The rest of the paper is organized as follows. Section 2 describes the system models. Section 3 verifies the model proposed by using simulation results. Finally, Section 4 summarizes the conclusions and described further work required.

\section{System Model}

This research simulates a single macro cell in a cellular networks system, implementing some user devices to establish D2D communication. The simulations investigate the implementation of power control methods in both types of transmission, i.e. downlink and uplink transmissions used within D2D communication's underlying macro cell coverage. The simulation assumes also that D2D communica- tions uses the same frequency spectrum as is used in the macro cell. Therefore, interference between each devices and the base station should be the main problem encountered.

In the first scenario, the simulation investigates the problem of interference caused DL signal sent from D2D UE (D2DT) to the macro cell's receiver MUE. A number of D2D communications will interfere with the MUE that is being served by the macro cell. The SINR distribution of MUE will be analyzed. This first scenario is shown in Fig. 1a. The second scenario analyzes the impact of interference on the receiver of the macro cell, caused by uplink interference of the devices, as presented in Fig. 1b.

For the first scenario, let $P_{T-M}$ be the transmit power of the macro cell. Then, $P_{R \_}^{D L}{ }^{L}$ is is the power received at the receiver side (at the MUE that desires to receive the of the signal) can be formulated as [13]:

$$
P_{R \_M U E}^{D L}=P_{T \_M} \cdot G_{T \_M},
$$

where $P_{T_{-} M}$ is the transmit power of the macro cell and $G_{T \_}$is the channel gain from the macro cell to MUE. For simulating $G_{T_{-} M}$, the Eq. (1) can be used as:

$$
G_{T \perp}=P L_{M} \cdot h_{M},
$$

where $P L_{M}$ is the propagation loss between the macro cell and MUE, and $h_{M}$ is the channel's small scale fading factor from the macro cell to MUE. However, this research does not take into account the effect of small scale fading. Therefore, it characterizes only the propagation loss caused by the distance between the transmitter and the receiver. In this system, $P_{T \_} . G_{T \_}$means the received signal power at the distance $d$. Since this paper does not consider the ef- 
fect of small scale fading, $G_{T \_} M$ could be denoted as a propagation model. This paper adopts the propagation model formulated in [9] and [15].

This formula describes the path-loss link between pieces of user equipment, which calculates the propagation loss from D2D UE/D2DT of D2DR and MUE to the macro cell or vice versa:

$$
P L_{M}[\mathrm{~dB}]=128.1+37.6 \log d[\mathrm{~km}]
$$

and this equation reflects D2D links and calculates the link propagation level between the transmitter of D2D UE (D2DT) and the receiver of D2D UE (D2DR):

$$
P l_{D 2 D}[\mathrm{~dB}]=148+40 \log d[\mathrm{~km}],
$$

where $P l_{D 2 D}$ is the propagation loss from UE (D2D equipment or MUE) to the macro cell or vice versa), $P l_{D 2 D}$ is propagation loss from $\mathrm{D} 2 \mathrm{D}$ communication pairs between D2DT and D2DR, and $d$ is the distance between the transmitter and the receiver in $\mathrm{km}$.

In a DL transmission, the signal quality of MUE is measured by SINR:

$$
\operatorname{SINR}_{M U E_{1}, M}^{D L}=\frac{P_{R \perp M U E}^{D L}}{r d(k) P_{D 2 D T_{k}, M U E_{1}} \cdot G_{D 2 D T_{k}, M U E_{1}}+N},
$$

where $S I N R_{M U E_{1}}$ is the SINR values on MUE with the signal received from the macro cell. $P_{R \_}^{D L}{ }_{-}$is the received power at MUE for the desired signal from the macro cell. $r d(k)$ is the equality indicator used for downlink resources, 0 and 1 are for the different and same resource used, respectively [13]. $G_{D 2 D T_{k}, M U E_{1}}$ is the channel gain between D2DT that interferes and MUE, and $N$ is the power of system noise.

$P_{R \perp M E}^{D L}$ can be calculated using Eq. (1) and the interference from $D 2 D R_{K}$ that uses the same frequency resource as the cellular networks can be formulated using Eq. (1), by replacing eNB indices with those for the transmitter, and the MUE1 indices remain the same as those of the receiver serving as the terminal under observation. As mentioned earlier, this paper does not consider the small scale fading factor and the same assumption applies to the link between D2DT and MUE.

In UL, SINR measured on eNB can be calculated as:

$$
\operatorname{SINR}_{e N B_{1}, M}^{U L}=\frac{P_{R_{-} e N B}^{U L}}{r d(k) P_{D 2 D R_{k}, M U E_{1}} \cdot G_{D 2 D R_{k}, M U E_{1}}+N},
$$

where $S I N R_{M U B_{1}}$ is the SINR values on eNB receiver with the signal received from the MUE transmitter. $P_{R \_M U E}^{U L}$ is the received power at eNB. $G_{D 2 D R_{k}, M U E_{1}}$ is the channel gain between $\mathrm{D} 2 \mathrm{D}$ and $N$ is the power of system noise.

\subsection{Power Control}

The power control method is implemented on both sides: D2D and the cellular network. This paper considers interference-related problems affecting cellular communication first. In the case of D2D communication, all equations presented in the following descriptions apply, with the indices replaced accordingly.

In order to reduce interference, the transmit power of the desired transmitter, i.e. eNB in this case, may be adjusted. The transmit power of the eNB macro cell, $P_{T-M}$ is established in the numerator of Eq. (5). The transmit power of the desired transmitter, at the frame transmission time, $P_{T_{-} M}\left(t_{i}\right)$ will be adjusted at the next time of frame transmission, producing a new transmit power value $P_{T-M}\left(t_{i+1}\right)$. This paper uses $\gamma$ as a parameter of the TX power control method change occurring at the next frame transmission and based on the estimated value of current time of $\operatorname{SINR}\left(\operatorname{SINR}_{\text {est }}\left(t_{i}\right)\right) \cdot \operatorname{SINR}_{\text {est }}\left(t_{i}\right)$ will be compared with the predetermined SINR $R_{\text {tar }}$ value. Based on this argument, this paper introduces a $k$ parameter, to indicate whether the value of $\gamma$ will increase the transmit power or decrease the transmit power or whether the same transmit power will be kept for the next time of frame transmission. The general expression of $P_{T \_}\left(t_{i+1}\right)$ can be written as:

$$
P_{T \_M}\left(t_{i+1}\right)=P_{T \_M}\left(t_{i}\right)+k \cdot \gamma .
$$

The value of $k$ will be determined according to the $\operatorname{SINR}\left(t_{i}\right)$. In this case, there are three likely conditions of $\operatorname{SINR}_{\text {est }}\left(\mathrm{t}_{i}\right)$ [13]:

- first, if $S I N R_{\text {est }}$ is smaller than $S I N R_{\text {tar }}$, then $k$ will be a positive value,

- second, if $S I N R_{\text {est }}$ is same as $S I N R_{\text {tar }}$, then $k$ will be equal to 0 ,

- third, if $S I N R_{e s t}$ is greater than $S I N R_{t a r}$, then $k$ will be a negative value.

The exact values of $k$ and $\gamma$ depend on the power control method that is going to be applied. This paper uses two power control methods: AC1 and AC2. The manner in which the values of $k$ and $\gamma$ impact the two power control methods is explained later on in this section.

And then, another scenario has to be met by the power control method. The value of $P_{T-M}\left(t_{i+1}\right)$ in Eq. (7) must not exceed the value of maximum and must not be lower the minimum transmit power of eNB, i.e. $P_{\max }$ and $P_{\min }$, respectively. Then, the final value of $P_{T \_}\left(t_{i+1}\right)$ at the next frame transmission using the output of the power control method will be determined based on the expression below. To avoid the confusion, $P_{T \_}\left(t_{i+1}\right)$ that is formulated in Eq. (7) is re-denoted as $P_{T-M}\left(t_{i+1}\right)$ :

$$
\begin{aligned}
& P_{T \_M}\left(t_{i+1}\right) \\
= & \begin{cases}\min \left\{P_{T \_M}^{*}\left(t_{i+1}\right), P_{\max }\right\} & \text { if } \operatorname{SINR}_{\text {est }}\left(t_{i}\right)<S I N R_{\text {tar }} \\
P_{T \_M}^{*}\left(t_{i+1}\right) & \text { if } \operatorname{SINR}_{\text {est }}\left(t_{i}\right)=S I N R_{\text {tar }} . \\
\max \left\{P_{T \_M}^{*}\left(t_{i+1}\right), P_{\min }\right\} & \text { if } \operatorname{SINR}_{\text {est }}\left(t_{i}\right)>S I N R_{\text {tar }}\end{cases}
\end{aligned}
$$

Equation (8) will guarantee that the power output of the power control methods will be within the permitted transmit power limits of UE (MUE and D2DT) and eNB. 


\subsection{AC1 Power Control}

The AC1 power scheme procedure is simple and can be implemented easily in practice. As in [13], the paper has applied this power control method to a two-tier heterogeneous (femto-cell and macro cell) network. AC1 uses a fixed value for multiplication of $k$ and $\gamma$. The value of $\gamma$ is set to be a constant and is a simulation parameter. The value of $k$, in turn, depend on the values of $\operatorname{SINR}_{\text {est }}\left(t_{i}\right)$, as explained earlier, and can be expressed as:

$$
k=\left\{\begin{array}{rl}
+2 & \text { if } \operatorname{SINR}_{\text {est }}\left(t_{i}\right)<S I N R_{\text {tar }} \\
0 & \text { if } \operatorname{SINR}_{\text {est }}\left(t_{i}\right)=S I N R_{\text {tar }} \\
-2 & \text { if } \operatorname{SINR}_{\text {est }}\left(t_{i}\right)>S I N R_{\text {tar }}
\end{array} .\right.
$$

\subsection{AC2 Power Control}

This research uses the different values of multiplication between $k$ and $\gamma$ in order to increase or decrease the transmit power in the AC2 power control method:

$$
k=\left\{\begin{array}{rl}
+3 & \text { if } \operatorname{SINR}_{\text {est }}\left(t_{i}\right)<S I N R_{\text {tar }} \\
0 & \text { if } \operatorname{SINR}_{\text {est }}\left(t_{i}\right)=S I N R_{\text {tar }} \\
-3 & \text { if } \operatorname{SINR}_{\text {est }}\left(t_{i}\right)>S I N R_{\text {tar }}
\end{array},\right.
$$

The different values of $k$ when $\operatorname{SINR}_{\text {est }}\left(t_{i}\right)<\operatorname{SINR} R_{\text {tar }}$ and $S I N R_{\text {est }}\left(t_{i}\right)>S I N R_{\text {tar }}$ are intended to affect the controlled transmit power when it is increased or decreased.

The $\gamma$ is based on the average received interference power at the observed terminal (D2D UE). Calculation of the average interference power is based on the moving average method, as illustrated in Fig. 1a-b. The shift register is used to store the interference power detected at the observed terminal (MUE or eNB). Then, the average value of interference power is:

$$
\bar{I}=\frac{1}{n} \sum_{i=1}^{n} I_{i} .
$$

Then, $\gamma$ is calculated:

$$
\gamma=\left|P_{T M}\left(t_{i}\right)-\bar{I}\right| .
$$

SINR values at the MUE for DL and eNB for UL transmission are analyzed through simulation.

\section{Simulation Results}

This paper presents some simulations investigating interference management by considering D2D communication in an underlying cellular network BS. A single macro cell is considered with the position of eNB base station at the center of the macro cell network, as discussed earlier. This simulation is set up to randomize the location of D2D devices 10 times in each simulation, throughout the macro cell network's coverage.

Table 1 shows the values of simulation parameters. The maximum and minimum transmit power of eNB and D2D equipment are set to $46 \mathrm{dBm}$ and $26 \mathrm{dBm}$, respectively. The macro cell radius of eNB is set up to $900 \mathrm{~m}$, which is
Table 1

Simulation parameters

\begin{tabular}{|l|c|}
\hline \multicolumn{1}{|c|}{ Parameter } & Value \\
\hline \hline Number of macro cell networks & 1 \\
\hline Macro cell network radius & $1000 \mathrm{~m}$ \\
\hline Radius DUE (D2DT to D2DR) & $100 \mathrm{~m}$ \\
\hline Macro cell TX power (maximum) & $46 \mathrm{dBm}$ \\
\hline UE TX power (maximum) & $26 \mathrm{dBm}$ \\
\hline Frequency carrier & $1800 \mathrm{MHz}$ \\
\hline Antenna pattern & Omni-directional \\
\hline Channel bandwidth & $10 \mathrm{MHz}$ \\
\hline Number of transmitters & 1 \\
\hline Number of receivers & 1 \\
\hline White noise spectral density & $-174 \mathrm{dBm} / \mathrm{Hz}$ \\
\hline Radius MUE to macro cell & 0 s.d. $600 \mathrm{~m}$ \\
\hline Radius D2DT to macro cell & 600 s.d. $900 \mathrm{~m}$ \\
\hline
\end{tabular}

typical of a cellular network in an urban area. In a cell edge situation, UL transmit power will be a big problem if each device uses the same frequency band, since devices that are far away from its pair will maximize the power level to maintain the communication link.

This simulation divides the coverage of the macro cell into two areas, cell center $(0-600 \mathrm{~m})$ and cell edge (600-900 m). The distance is measured from the base station eNB. For evaluating interference in DL and UL transmissions in this paper, both MUE and D2D devices pairs are randomly deployed at the edge of the cell $(600-900 \mathrm{~m})$. The system bandwidth is set to $10 \mathrm{MHz}$ and the system noise is set to $-174 \mathrm{dBm} / \mathrm{Hz}$. The value of $\gamma$ for $\mathrm{AC} 1$ is set to be $2 \mathrm{~dB}$, and the SINR target is set to $0 \mathrm{~dB}$, which corresponds to a SINR value typical for data traffic. Note that these experiments used software simulation and were repeated for 10 times. The average simulation results are shown in the graph.

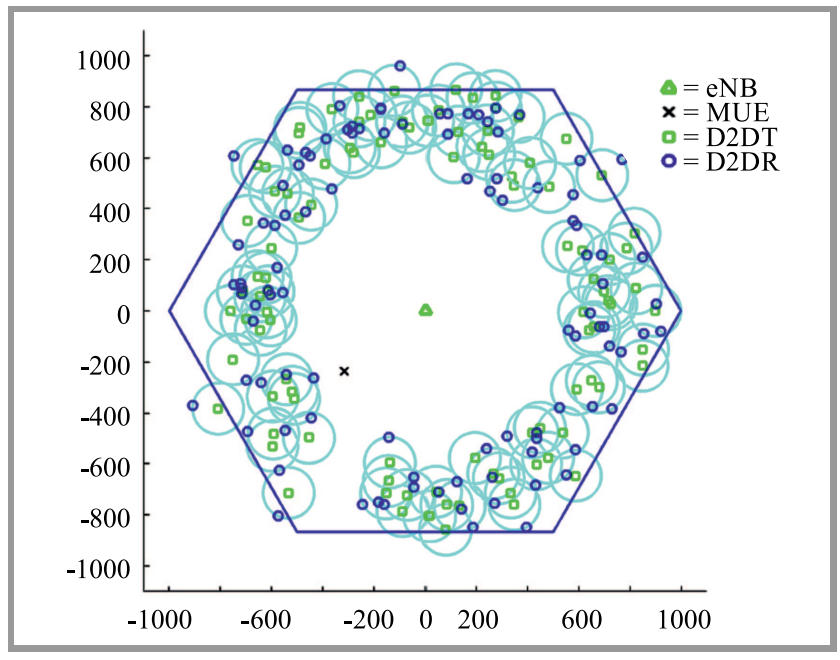

Fig. 2. Simulations of a single cellular network (eNB) with D2D deployment. 
The first scenario investigates the power control scheme to manage SINR in a DL transmission. In this case, eNB in the center of a macro cell sends the signal to the MUE device, while D2D equipment (as D2DT) also transmits the signal to another piece of D2D equipment (D2DR). So, the transmission signal from D2DT will interfere with MUE. This scenario uses QoS parameters, such as level of SINR in the receiver part, measured on MUE devices in DL [16].

The second scenario involved an UL transmission, where MUE transmitted an UL signal to eNB, while D2DR equipment also transmitted a signal to D2DT equipment. So, the D2DR transmission signal interfered with the MUE signal uplink to eNB. This scheme utilized the same QoS parameters as used on the base station eNB in the first scheme.

Figure 2 shows the simulation scenario using a cellular network system with an eNB as a macro cell, MUE equipment and the number of D2D pairs increased to 100 devices. In a DL transmission, as shown in Fig. 3, the increased number of pieces of D2D equipment could decrease the SYSTEM's performance. With UE only, SINR values achieved approximately $30 \mathrm{~dB}$ for every power transmit method, without $\mathrm{AC} 1$ or $\mathrm{AC} 2$. If the number of $\mathrm{D} 2 \mathrm{D}$ pairs was increased to 50, SINR dropped to about $25.5 \mathrm{~dB}, 25 \mathrm{~dB}$, and 24 for $\mathrm{AC} 1, \mathrm{AC} 2$, and without power control (PC) or fixed power level (FC), respectively.

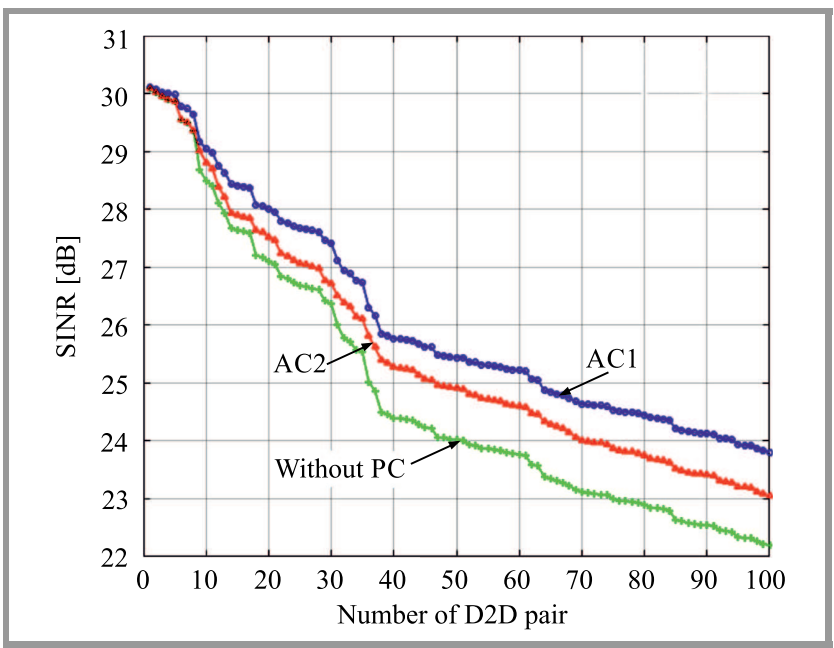

Fig. 3. SINR downlink values measured in a MUE as the number of increased D2D pairs, under a single cell scenario of a macro cell network without power control and with adaptive power control.

Figure 4 shows the result of UL transmission simulation. In this result, the increased number of pieces of D2D equipment also could decrease the system's performance. It can be noticed in Fig. 4 that when there is no interference at MUE, SINR achieves up to $18 \mathrm{~dB}$ for systems without $\mathrm{PC}$, with $\mathrm{AC} 1$ and $\mathrm{AC} 2$ methods, respectively. When the number of D2D pairs equals 50 devices, SINR drops to about $2 \mathrm{~dB}$ and less than 0 when $100 \mathrm{D} 2 \mathrm{D}$ pairs are used without PC. With $\mathrm{AC} 1$ and $\mathrm{AC} 2$ implemented, SINR

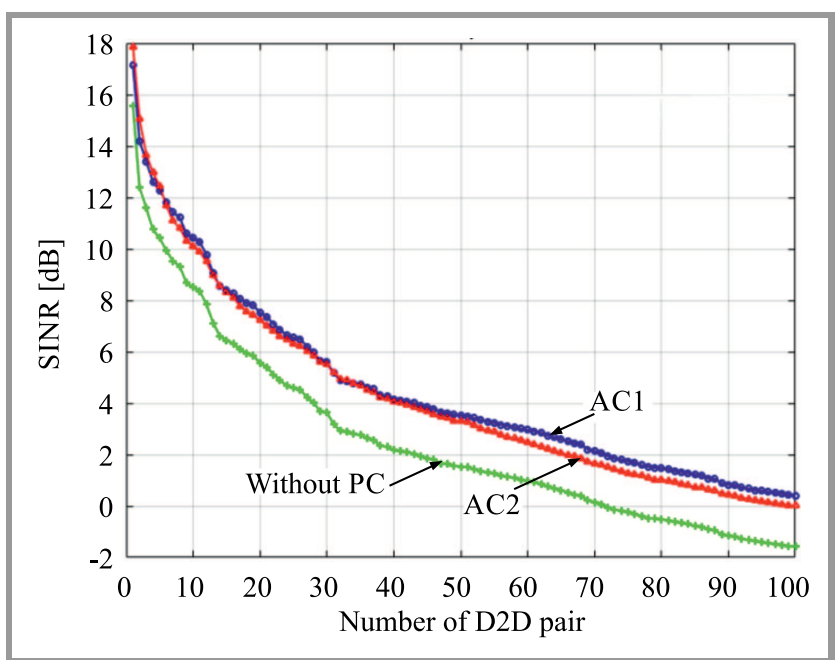

Fig. 4. SINR uplink values measured in eNB as the number of increased D2D pairs under a single cell scenario of a macro cell network with MUE, without power control and with AC1 and AC2 adaptive power control.

dropped to about $3.5 \mathrm{~dB}$ for both schemes and remained at above 0 when D2D communication setup involved 100 pairs within a macro cell network.

Based on those simulations, it can be noticed that AC1 and AC2 methods are capable of managing SINR performance, as shown in Figs. 3-4. These power control methods could work because the transmitter of each device adjusts the transmit power according to the estimated SINR and interference values. In summary, based on the result shown in Fig. 4, these power control methods can only manage up to $100 \mathrm{D} 2 \mathrm{D}$ pairs to ensure SINR level of up to $0 \mathrm{~dB}$.

\section{Conclusion}

This paper investigates different power control methods used to mitigate interference between two or more user devices, with the number of D2D pairs equaling up to 100 . The decision whether to increase or decrease the power level on BS or on the transmitter of the D2D pair is based on the estimated current SINR. The power control methods may be divided based on two aspects. The first of them is based on the fixed power level or uses no power control to manage the power level of the transmitter. The second uses adaptive power controls with two schemes (AC1 and $\mathrm{AC} 2$ ). Based on the measured SINR, the simulation results show that both power control methods contribute to managing SINR and network performance. AC1 and AC2 can improve SINR by up to $1 \mathrm{~dB}$ in each method compared to FC in both DL and UL transmissions.

\section{Acknowledgements}

The authors would like to thank Universitas Muhammadiyah Yogyakarta for supporting this research and Mr. Slamet Riyadi for his suggestions about this paper. 


\section{References}

[1] A. Gupta and R. K. Jha, "A survey of 5G network: Architecture and emerging technologies", IEEE Access, vol. 3, pp. 1206-1232, 2015 (doi: 10.1109/ACCESS.2015.2461602).

[2] R.-G. Cheng, N.-S. Chen, Y.-F. Chou, and Z. Becvar, "Offloading multiple mobile data contents through opportunistic device-todevice communications", Wirel. Personal Commun., vol. 84, no. 3, pp. 1963-1979, 2015 (doi: 10.1007/s11277-015-2492-1).

[3] P. Mach, Z. Becvar, and T. Vanek, "In-band device-to-device communication in ofdma cellular networks: A survey and challenges", IEEE Commun. Surveys \& Tutor., vol. 17, no. 4, pp. 1885-1922, 2015 (doi: 10.1109/COMST.2015.2447036).

[4] M. N. Tehrani, M. Uysal, and H. Yanikomeroglu, "Device-to-device communication in 5G cellular networks: Challenges, solutions, and future directions", IEEE Commun. Mag., vol. 52, no. 5, pp. 86-92, 2014 (doi: 10.1109/MCOM.2014.6815897).

[5] A. Asadi, Q. Wang, and V. Mancuso, "A survey on device-to-device communication in cellular networks", IEEE Commun. Surveys \& Tutor., vol. 16, no. 4, pp. 1801-1819, 2014 (doi: 10.1109/COMST.2014.2319555).

[6] L. Wei, R. Hu, Y. Qian, and G. Wu, "Enable device-to-device communications underlaying cellular networks: Challenges and research aspects", IEEE Commun. Mag., vol. 52, no. 6, pp. 90-96, 2014 (doi: 10.1109/MCOM.2014.6829950).

[7] S. T. Shah, S. F. Hasan, B.-C. Seet, P. H. J. Chong, and M. Y. Chung, "Device-to-device communications: A contemporary survey", Wirel. Personal Commun., vol. 98, no. 1, pp. 1247-1284, 2018 (doi: 10.1007/s11277-017-4918-4).

[8] R. Yin, C. Zhong, G. Yu, Z. Zhang, K. K. Wong, and X. Chen, "Joint spectrum and power allocation for D2D communications underlaying cellular networks", IEEE Trans. on Vehicular Technol., vol. 65, no. 4, pp. 2182-2195, 2016 (doi: 10.1109/TVT.2015.2424395).

[9] S. Y. Shin and T. A. Nugraha, "Cooperative water filling (CoopWF) algorithm for small cell networks", in Proc. Int. Conf. on ICT Convergence ICTC 2013, Jeju, South Korea, 2013, pp. 959-961 (doi: 10.1109/ICTC.2013.6675527).

[10] P. Mach and Z. Becvar, "Combined shared/dedicated resource allocation for device-to-device communication", arXiv preprint arXiv:1711.01866, 2017.

[11] N. Lee, X. Lin, J. G. Andrews, and R. W. Heath, "Power control for D2D underlaid cellular networks: Modeling, algorithms, and analysis", IEEE J. on Selec. Areas in Commun., vol. 33, no. 1, pp. 1-13, 2015 (doi: 10.1109/JSAC.2014.2369612).

[12] Y. Jiang, Q. Liu, F. Zheng, X. Gao, and X. You, "Energyefficient joint resource allocation and power control for D2D communications", IEEE Trans. on Vehicular Technol., vol. 65, no. 8, pp. 6119-6127, 2016 (doi: 10.1109/TVT.2015.2472995).

[13] M. Susanto, H. Fitriawan, A. Abadik, and Herlinawati, "On the reduction of interference effect using power control for device-todevice communication underlying cellular communication network", in Proc. Int. Conf. on Elec. Engin. and Comp. Sci. ICECOS 2017, Palembang, Indonesia, 2017, pp. 28-32 (doi: 10.1109/ICECOS.2017.8167149).

[14] A. Ramezani-Kebrya, M. Dong, B. Liang, G. Boudreau, and S. H. Seyedmehdi, "Joint power optimization for device-to-device communication in cellular networks with interference control", IEEE Trans. on Wirel. Commun., vol. 16, no. 8, pp. 5131-5146, 2017 (doi: 10.1109/TWC.2017.2706259).

[15] S. K. Pal, T. A. Nugraha, S. Shams, and A. Rahman, "Resource allocation strategy using optimal power control for mitigating twotier interference in heterogeneous networks", in Proc. Wirel. Commun. and Networking Conf. Worksh. WCNCW 2014, Istanbul, Turkey, 2014, pp. 104-109 (doi: 10.1109/WCNCW.2014.69348769).

[16] V. Tikhvinskiy, G. Bochechka, and A. Gryazev, "QoS requirements as factor of trust to 5G network", J. of Telecommun. and Inform. Technol., no. 1, pp. 3-8, 2016.

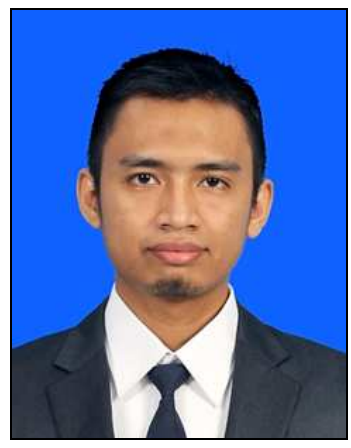

Toha Ardi Nugraha received his B.Sc. degree in Telecommunication Engineering from Telkom University, Indonesia, in 2011 and M.Sc. degree in IT Convergence Engineering from Kumoh National Institute of Technology, South Korea, in 2014. He joined the Department of Electrical Engineering, Universitas Muhammadiyah Yogyakarta, Indonesia, as a lecturer in 2016. He has worked at the R\&D Center PT Telkom Indonesia in 2009-2012 as a research assistant. His current research interests include wireless and mobile networks, D2D, Li-Fi, and IoT.

E-mail: toha@ft.umy.ac.id

Universitas Muhammadiyah Yogyakarta

Jl. Brawijaya, Bantul

Yogyakarta 55183, Indonesia

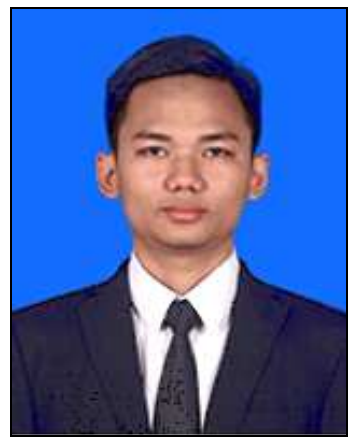

Muhammad Putra Pamungkas received his B.Sc. degree in Electrical Engineering from Universitas Muhamadiyah Yogyakarta, Indonesia, in 2017. He is currently pursuing M.Sc. degree at the Department of Electrical Engineering, Institut Teknologi Bandung, Indonesia, with a focus on telecommunications. His research interests include computer networking, mobile communications, wireless networks and D2D communications.

E-mail: putrapamungkas.15031995@gmail.com Universitas Muhammadiyah Yogyakarta

Jl. Brawijaya, Bantul Yogyakarta 55183, Indonesia

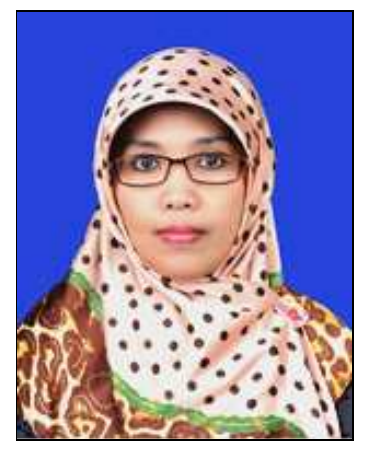

Anna Nur Nazilah Chamim received her B.Sc. degree in Electrical Engineering from Universitas Muhammadiyah Yogyakarta, Indonesia in 2001, and M.Sc. degree from the Department of Electrical Engineering, Universitas Gadjah Mada, Indonesia in 2014. Since 2009 she has worked as a Head of Department of Telecommunication Engineering Politeknik PPKP Yogyakarta. She also worked as a lecturer at the Department of Electrical Eng., Universitas Muhammadiyah Yogyakarta. Her research interests include telecommunications, digital signal and image processing.

E-mail: anna_nnc@umy.ac.id

Universitas Muhammadiyah Yogyakarta

Jl. Brawijaya, Bantul

Yogyakarta 55183, Indonesia 